DOI: $10.24044 /$ sph.2017.1.14

\title{
A STUDY ON THE EFFECTIVENESS OF EDUCATIONAL INTERACTIVE MULTIMEDIA INTEGRATION IN THE PROCESS OF FOREIGN LANGUAGE TEACHING
}

\author{
D. C. Baeva
}

\author{
Ph.D.,Doctor of Pedagogical Sciences \\ University of Ruse Angel Kanchev \\ Ruse, Bulgaria
}

\begin{abstract}
The information about the effectiveness of educational interactive multimedia on the foreign language teaching and its combination with other technical tools (blended learning) is still incomplete and insufficient in terms of phonemic students' awareness. Outlined tendency is learners' preference to cognitive rather than metacognitive strategies in the process of study. In the pursuit of knowledge, the paper presents an experiment related to interactive multimedia integration in teaching Bulgarian as foreign language at beginner's level, analyzes the results and draws out methodical conclusions. It is necessary to consider the psychological aspects which are important for learners' attitude to use computers in the process of foreign language learning.
\end{abstract}

Keywords: interactive multimedia; blended learning; phonemic awareness.

At present, the new frameworks for evaluating educational results gain a growing influence. Actually, they concentrate on activities leading to a faster adaptability to reality, look for results which put aside declarative cognition and foster the analytical and flexible educational resources. They are aiming to assist the development of learner's qualities like self-discipline, ability to communicate and collaborate, reflectiveness, etc. The frameworks applied cast the leading role to the educational results and focus on the teaching medium as a means for providing optimal conditions for communication and cooperation [4].

Many scholars admit that computer based learning helps learners acquire different skills as the program provides them with clear examples for using specific strategies. Computers have a significant role as an environment for developing both specific and general language and learning skills. Moreover, they do not just model skills - computers provide learners with tools not only for practising but for knowledge improvement as well [5].

The modern methodical principles require that the programs should be focused on the needs and specificities of learners who become the central figure in the process of software designing. Besides, suitable strategies have to be worked out to ensure the effective individual learning [2].

The design of teaching is a process by which the teacher chooses and determines the best methods in order to teach concrete learners in a concrete context. 
Based on psychological, linguistic and methodical data, this study aims to work out guides for integrating applied educational software tools in the process of teaching Bulgarian language to foreigners at beginner's level. The concrete practical task is to promote suitable specialized educational software. It would be achieved with the construction of appropriate medium for eliminating the specific educational difficulties.

The educational software goal is to enhance students' knowledge, assist the teaching process, and motivate students' independent learning. After due analysis, the author chose exercises from Разбирате ли Български? (Do you understand Bulgarian?) software, and used them for the purpose of this investigation. The elaboration is an applied software product designed for both in-class and out-of-class activities. It provides a platform for systematical and consistent development of audible culture for the Bulgarian language, without any restrictions for time and place, so that learners are able to practice their skills and experiment them at their own pace.

When choosing methodology for conducting the experiment for evaluating the effectiveness of the multimedia as an educational means, a number of factors should be considered such as: target specificity, principles of preciseness, proven high ethics, respect to discretion and effective combination of qualitative and quantitative indices and criteria.
To assess the educational effectiveness of the Do you understand Bulgarian software system it was necessary to find out an adequate evaluation framework with the help of welldefined process so as to generate meaningful and valid results. The stage of evaluating the designed teaching method imposed the following tasks:

- Adherence to the basic requirements for preparation, performance, assessment and interpretation of the results from the experiment.

- Performance of quantitative and qualitative analysis. The latter must be conducted not only on experimental empirical basis, but it should consider definite theoretical statements and find out the theoretical explanation of one or other result.

The experiment of this research included 28 persons - international students enrolled for the preparatory course in 2015 at the University of Ruse. To substantiate the extract representativeness, the analysis considered the evidence that the extract possesses statistical properties inherent to the general set, which makes the investigation valid for all who are learning Bulgarian as a foreign language.

The participants in the experiment were asked to answer an anonymous questionnaire that included items helping to extract objective data about processes, activities and individuals - the object of the inquiry.

- The first group of questions referred to gathering demographical and 
personal data: sex, nationality and mother tongue;

- The second group of questions were related to learners' motivation to use educational software in the course of Bulgarian learning;

- The third group provoked the retrieval of information about the applicability of the software system.

Three groups were assigned for the experiment - two from the preparatory training course and one of foreign students visiting Bulgaria on Erasmus Program. The first two administrative groups included only Turkish students, while the third one consisted of three people from Spain, one from Greece, one from Azerbaijan, two from Georgia, and two from Armenia. The reason for this group to be included was inspecting the functional capabilities of the created experimental method, its universality and perspective; do the same laws that apply to the Turkish apply to the others from the preparatory training groups.

The first group (A) consisted of Turkish students and the second (B), consisted of students with various nationalities were picked to be experimental, while the third was for sample control.

Data and results processing was carried out on the basis of investigation, examination and analysis of documents and related scientific literature; searches in the web and e-databases; input and processing data from the observations and questionnaires; analysis of the results. The paper presents some interesting outcomes and the corresponding conclusions. They set up the option, after analysis of the qualitative and quantitative data, to draw out inferences and conclusions, which can be accepted valid for most users learning Bulgarian as a foreign language.

One of the ways to raise the effectiveness of contemporary foreign language teaching is the systematic involvement of various digital tools. At the same time, many teachers and instructors of foreign language teaching are looking for better visual and audible aids to assist the language teaching. In most cases, the technical aids (computers, in particular) are used non-systematically, in unorganized manner, with no preparation, clear targets and principles, which is to the detriment of the didactic documentation.

Yet, with the first attempts to integrate computers as additional obligatory mediator in the teaching process, the reactions caused a prolonged discussion in response to learners' adjustments that have changed for the last several years. At first, the enthusiasm arose in correspondence with the technical progress resources, but over the last decades the opinions became contradictory: on one hand, the attitude to ICT application is implicit, on the other hand - learners express quite an explicit attitude to computers as a second mediator. The questionnaire that was used for the purpose of this study included an item that analyzed learners' adjustments. The responders were asked if, unaided, they would use educational software for learning Bulgarian language. They gave the following answers: Yes, I would use - 
$68 \%$; No, I don't think it is necessary - $7 \%$; No personal opinion - $25 \%$.

The result is truly interesting and provocative for analysis, as the exclusively low percentage of the responders who think it is not necessary to use computer machines in the process of foreign language teaching shows that the potential users of the software system are either motivated or insufficiently interested, or uninformed to have their own opinion. Hence, the attention has to be directed to the systematic application of interactive software products so that to help learners estimate the benefits of the digital tools. In result, the foreign language teaching shall be closer to the educational standards of the developed countries that would correspond to EC directives for cultural collaboration.
The diagrams show a shift towards increased interest in the foreign language blended learning. In a century of total computerization regardless of the initial adjustments for traditional attitude to classical teaching methods, the opportunity to make use of personal computer benefits raises the interest in foreign language learning.

The respondents were also asked to answer the following question: Is your comprehension of Bulgarian language easier after the exercises? Certainly, the reply presumes a systematic application of the software proposed, but in this case the question gives a chance to evaluate the general adjustment regarding its usefulness. The results are encouraging: $68 \%$ claim "Yes, it is easier", $11 \%$ - "No, it is not", $21 \%-$ "No personal opinion" (see Fig. 2).
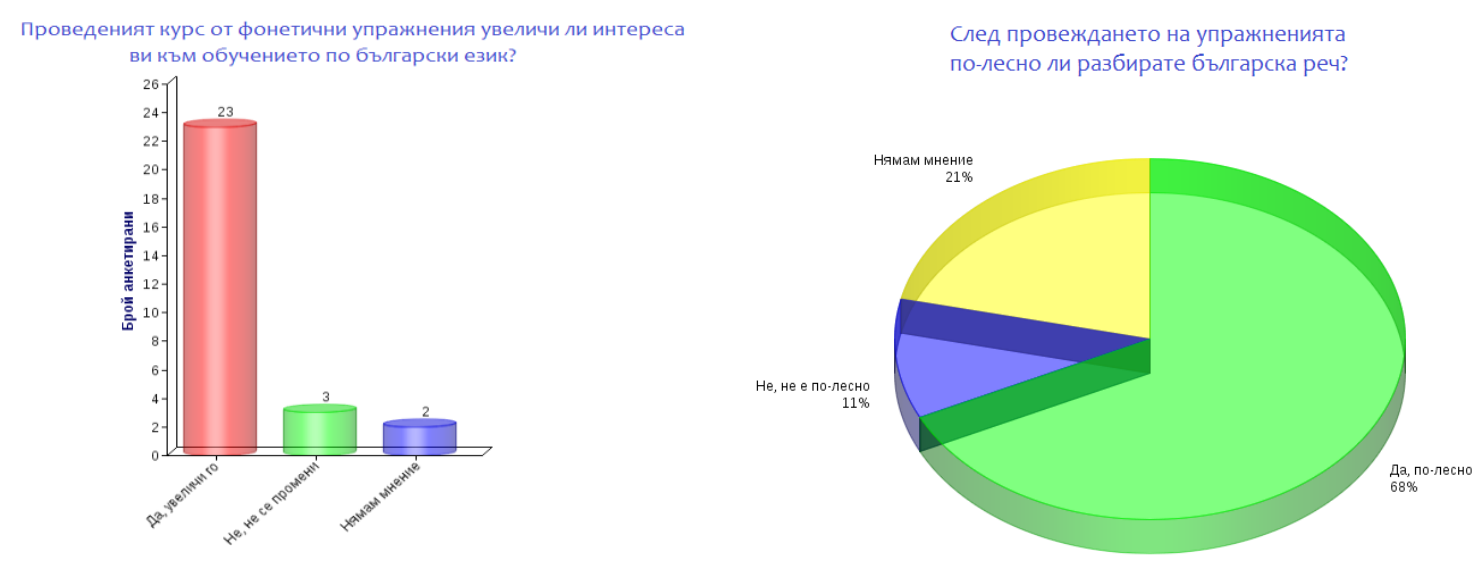

Figure 1. Explicit interest in phonetic exercises for learning Bulgarian as a foreign language

Figure 2. Opinions related to the benefits of listening exercises in foreign language teaching

The above mentioned results give us grounds to conclude that the appli- cation of multimedia software product enhances learners' interest to master 
the language system and motivate their study.

The question "Do you consider that the course adds to your acquisition of Bulgarian language?" aims to evaluate the adjustment of the international students for integrating applied software tools (the proposed software product in particular) in the process of learning Bulgarian language. Respondents' opinions will outline to a great extent the application domain of the web based course "Do you understand Bulgarian". The questionnaires contain a solid answer: "Yes, I do" $93 \%$, "No personal opinion" - $7 \%$ and nobody has answered that the course was useless.

The results from answering the question, "Would you recommend the application of educational software to other people learning Bulgarian language?" do certify the quality of teaching assisted with multimedia software tools. The high percentage of respondents' affirming answer - 96,4 \%, support the idea that education with integrated tools of suitable multimedia software shall motivate learners and shall intensify foreign language teaching.

In summary, all respondents participated with responsibility and sympa- thy in the experiment, clearly manifesting their willingness to work with the proposed multimedia software system of exercises that has been designed for developing phonemic awareness and independent practicing.

In order to find out how much the results of the work groups are reliable, if the proposed interactive system of exercises for hearing distinction and identification of Bulgarian phonemes in advanced stage of studying, we conducted a speech practice and postexperimental observatory period,

Two weeks after the training for a couple of hours the researched people were invited in a computer lab and without realizing the confidentiality of the lesson they did the interactive system of exercises. Talking sessions were also conducted. The willingness of the students for the implementation of the computer as a medium in the studying process is shown in the answers given to the question "Do you find beneficial the use of foreign language learning software in the process of studying in the future?"

Comparing the attained results it is visible that the inner motivation of the experimental groups has increased, reaching $100 \%$ approval. 


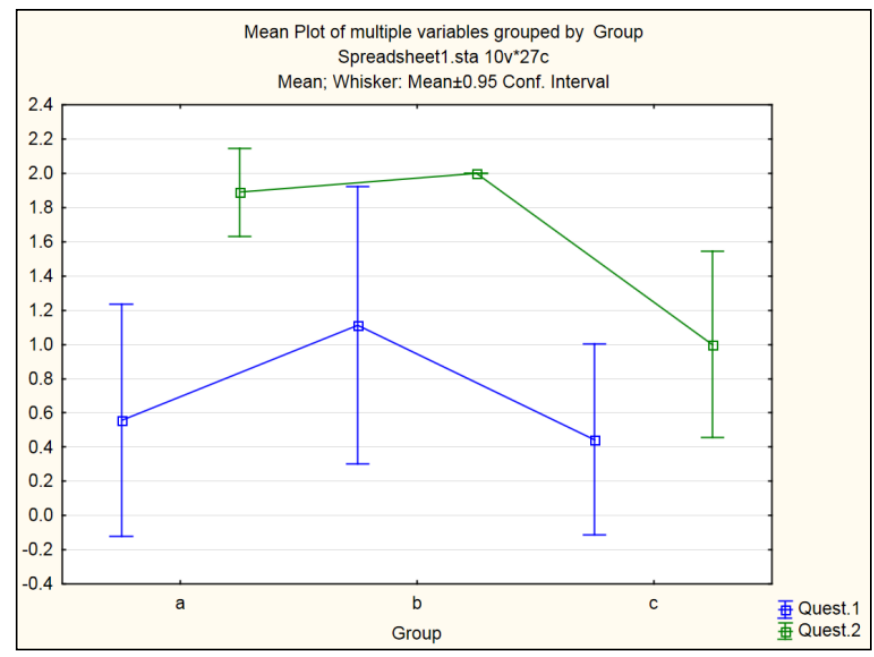

Figure 3. Influence of the interaction between the two factors on the inner motivation

It is clear, that the students from the control group in comparison with the experimental group tend to make up to 3,5 times more phonological mistakes. The results from the additional inspections showed, that the formed skills in the foreign students to hear different sounds in words and to relate them to a phoneme in Bulgarian, to comprehend the wordbuilding and meaningdistinguishing role of the phonemes are enough sustainable.

The following conclusions and ideas can be drawn out:

1. Foreign language teaching needs relevant conditions, which have to be provided by the educational institutions, for the purpose of using specialized software products or websites including sets of exercises for all levels of language acquisition.

2. The outlined tendency is learners' preference to cognitive rather than metacognitive strategies in the process of study. This estimation stimulates teachers to develop computerized models which focus on strategy diversification in order to enhance learners' motivation and hence, their achievements.

\section{Bibliography}

1. Baeva D. The interactive multimedia as a language learning resource on the initial level of language acquisition, Paradigmata poznání, 2014.

2. Chapelle C. The Relationship Between Second Language Acquisition Theory and Computer-Assisted Language Learning, The Modern Language Journal 2000.

3. Kehoe, Pitt 2006: Kehoe, A., I. Pitt., $24^{\text {th }}$ annual ACM international conference on Desihn of communication, Myrtle Beach, USA ACM.

4. Mayes, T. Designing Help Topics for use with Text-to-Speech \& de Freitas, S. Review of e-learning frameworks, models and theories. JISC e-learning models for desk study. Retrieved, December 22, 2006. 
5. Pennington, M.C., The road ahead. A forward-looking view of computers in language learning, CALL. vol. 4:3-19, 1996.

6. Warschauer, M. Online learning in second language classrooms: An ethnographic study', Cambridge University Press, 41-58, 2000.

(C) Baeva D. C., 2017. 